\title{
VEGETATION AS THE BIOINDICATOR OF HUMAN-INDUCED DEGRADATION IN KARST LANDSCAPE: CASE STUDY OF WASTE-FILLED DOLINES
}

\author{
VEGETACIJA KOT BIOINDIKATOR ANTROPOGENE \\ DEGRADACIJE KRAŠKE POKRAJINE: PRIMER Z ODPADKI \\ ZAPOLNJENE VRTAČE
}

\author{
Mateja BREG VALJAVEC ${ }^{1 *}$, Daniela RIBEIRO ${ }^{1}$ \& Andraž ČARNI ${ }^{2,3}$
}

\begin{abstract}
UDC 913:581.55(497.47)

581.55:551.435.82:628.472.2(497.47)

Mateja Breg Valjavec, Daniela Ribeiro \& Andraž Čarni: Vegetation as the bioindicator of human-induced degradation in karst landscape: case study of waste-filled dolines

Mismanagement practices, such as unsustainable waste disposal, created many degraded sites. In karst landscapes, old uncontrolled landfills are often located in dolines (sinkholes). Buried waste material in dolines represents degradation of landform, habitats and a potential danger of groundwater pollution. Buried waste provides heterogeneous ecological conditions on the surface, thus plant communities or individual plant species that developed on the surface of landfills can be used as a bioindicators of waste-filled doline sites and therefore indicators of land degradation. We aimed to discover the potential of vegetation to detect unknown locations of old dumpsites in suffusion dolines in Logaško polje (Dinaric Karst, Slovenia), either by plant communities or by plant species. We aimed to ascertain whether vegetation can indicate the dumping period by estimation of community succession stage. Locations and the age of waste-filled dolines (doline-dumps) were preliminary identified by a historical landscape study. Thus, we used time series of aerial photographs and digital photogrammetry tools for 3D modelling of historical terrain. Ecological evaluation was based on sampling the floristic composition of plots $(5 \times 5 \mathrm{~m})$. We analysed ecological conditions by Ellenberg bioindicator values, structure by life history traits and naturalness by hemerobic levels of plants. We studied in detail 30 up to 50-years-old waste-filled dolines that are interspersed by dry and mesic grasslands. Ecological evaluation demonstrated that the main driver of ecological diversity at doline-dumps is the time at which the doline was backfilled and succession started. Annual and eutrophic communities dominate the youngest doline-dumps, middle aged doline-dumps are covered by ni-
\end{abstract}

Izvleček

UDK 913:581.55(497.47)

Mateja Breg Valjavec, Daniela Ribeiro \& Andraž Čarni: Vegetacija kot bioindikator antropogene degradacije kraške pokrajine: primer $z$ odpadki zapolnjene vrtače

Slabe prakse upravljanja, kot je netrajnostno odlaganje odpadkov, so povzročile nastanek številnih degradiranih območij. V kraških pokrajinah, so tak primer stara odlagališča odpadkov v vrtačah. Z odpadki zasute vrtače predstavljajo degradacijo reliefne oblike, habitatov in potencialno nevarnost za onesnaženje podzemne vode. V vrtačah odloženi odpadki povzročajo heterogene ekološke razmere in niše za razvoj rastlinskih združb in posameznih rastlinskih vrst. Tako združbe kakor vrste je možno uporabiti kot bioindikatorje za odkrivanje z odpadki zasutih vrtač in antropogene degradacije v pokrajini. Namen prispevka je določiti potencial vegetacije (rastlinskih združb ali rastlinskih vrst) za odkrivanje degradacije na primeru neznanih odlagališč odpadkov v sufozijskih vrtačah Logaškega polja (Dinarski kras, Slovenija). Ugotavljali smo, ali lahko stopnja sukcesije določi obdobje degradacije. Lokacije in starost $\mathrm{z}$ odpadki napolnjenih vrtač so bile najprej določene s historičnogeografsko analizo. Uporabili smo zgodovinske aerofotogrametrične posnetke, metode digitalne fotogrametrije in $3 \mathrm{D}$ modeliranje zgodovinskega reliefa. Ekološka ocena degradiranih vrtač je določena na osnovi floristične sestave vzorčnih ploskev $(5 \times 5 \mathrm{~m})$, in sicer s pomočjo Ellenbergovih bioindikatorskih vrednosti, strukture $\mathrm{z}$ življenjskimi oblikami rastlin in njihovimi funkcionalnimi znaki ter stopnjah hemerobije (naravnosti) rastlin. Podrobneje smo preučili vzorec $30 \mathrm{z}$ odpadki zapolnjenih vrtač (v zadnjih 50 letih). Slednje so v pokrajini nerazpoznavne, preraščene s raznoliko vegetacijo znotraj travnikov. Ekološka ocena je pokazala, da je glavno gonilo ekološke raznolikosti na degradiranih vrtačah čas v katerem je bila vrtača zapolnjena. Enoletne in evtrofne združbe prevladujejo na najmlajših odlagališčih, na srednje

\footnotetext{
${ }^{1}$ Research Center of the Slovenian Academy of Sciences and Arts, Anton Melik Geographical Institute, Gosposka ulica 13, 1000 Ljubljana, Slovenia. e-mail: mateja.breg@zrc-sazu.si

${ }^{2}$ Research Center of the Slovenian Academy of Sciences and Arts, Jovan Hadži Institute of Biology, Novi trg 5, 1000 Ljubljana, Slovenia.

${ }^{3}$ University of Nova Gorica, Vipavska cesta 13, 5000 Nova Gorica, Slovenia

Received/Prejeto: 03.10.2016
} 
trophilous perennial forbs and, finally, communities developed towards mesic grassland. We conclude that plant communities in combination with diagnostic plant species can be used as a bioindicators of doline-dumps in agricultural landscape of Logaško polje and can therefore indicate the sites of potential groundwater pollution sources but not the type of long-time buried waste.

Key words: suffusion dolines, plant communities, waste dumping, Ellenberg indicator values, polje. starih odlagališčih smo zaznali nitrofilne trajnice in najbolj razvite združbe na gojenih mezofilnih travnikih, ki so zadnji stadij travniške sukcesije. Dokazali smo, da so rastlinske združbe $\mathrm{v}$ povezavi $\mathrm{z}$ diagnostičnimi rastlinskimi vrstami, uporabne kot bioindikatorji za določanje lokacij starih (do 50 let) in recentnih degradiranih vrtač v kmetijski pokrajini kraškega polja. Z uporabljeno metodo lahko posredno odkrivamo potencialne vire onesnaženja kraške vode, ni pa metoda dovolj preizkušena za opredeljevanje tipa podzemno odloženih odpadkov.

Ključne besede: sufozijske vrtače, rastlinske združbe, odlaganje odpadkov, Ellenbergove indikatorske vrednosti, kraško polje.

\section{INTRODUCTION}

Dolines are the most common, typical and representative landform (Sauro 2003), even more they are considered as the diagnostic karst landform (Ford \& Williams 1989, 2007) of karst landscapes. According to this, in conservation policy more attention should be paid to the geodiversity potential of unique landforms (Smrekar et al. 2016), habitat biodiversity and conservation. From geographic point of view, dolines can be considered as unique and individual geotop (Giusti 2016) with microclimate characteristics (e.g. thermal inversion) (Gams 2003) that induce specific vegetation patterns which can be called landform-conditioned vegetation patterns or landform-vegetation units (Baroni et al. 2007). The landform-vegetation units express consistent correlations among landforms, geomorphic processes and diagnostic plant communities. These communities act as phytoindicators of specific geomorphologic units derived from the activity of a single geomorphic process or from a combination of different geomorphic processes (Baroni et al. 2007).

The species composition of the herb layer of the dolines is markedly different than in surrounding grasslands (Bátori et al. 2009), so doline could be recognized as an individual landform-vegetation unit. In Central Europe dolines are considered as potential refugia for many vascular plants under upcoming global warming and are thus very valuable from a nature conservation point of view (Bátori et al. 2014). Dolines can be considered as the link between karst surface and underground, especially if we consider their role in karst processes (Gams 2000) and genesis (e.g. the theory of denuded caves by Mihevc et al. 1998). The direct connection between the surface and the underlying high permeability aquifers makes karst aquifers extremely vulnerable to pollution (Gutiérrez et al. 2014). Besides, karst underground is a unique habitat for many protected and endemic species (Pipan \& Culver 2013; Fišer et al. 2014) and the quality of underground abiotic and biotic sphere is the result of ecological conditions on the karst surface.

Despite the high coverage of carbonate rocks and the economic importance of karst areas in Slovenia, current standards for karst protection are loosely defined (Ravbar \& Šebela 2015) and human-induced degradation is still in progress. Human-induced filling of the dolines is considered as degradation of typical karst landscape from the perspective of typical geomorphology and landform. However, if we look from the perspective of the farmer or agricultural scientists, filling of dolines (if the material were natural and non-toxic, such as rocks and gravel of different size) could be considered as a positive measure to ensure more land of greater quality that enables the usage of modern machines etc. It can be compared to other agricultural land improvements like cultural terraces that are in Slovenia very common (Šmid Hribar et al. 2017; Kladnik et al. 2017).

Nevertheless, human activities may lead to a great variety of impacts, including degradation of the karst landscape, destruction of the epikarst and loss of karst landforms (Gutiérrez et al. 2014). Old pollution sources in a form of waste-filled dolines are of great concern due to the fact that waste material may remain active for many decades and even hundreds of years, depending on the physical conditions. Vegetation development is disturbed depending on the type of buried waste and remediation used. Gutiérrez et al. (2014) pointed out that illegal waste dumping and infiltration of liquid waste from landfills have effects on 1) water quality deterioration; 2) changes in karst landscape; 3) ecosystem contamination, and 4) extinction of rare species. Some studies on doline degradation in Dinaric Karst landscapes were done in regard to uncontrolled waste dumping and landfilling (Breg 2007; Cernatič Gregorič \& Zega 2010; Kovačič \& Ravbar 2013; Breg Valjavec 2014) and the impacts were evaluated from various perspectives, such as hydrology 
(Kogovšek \& Petrič 2013) or topography (Breg Valjavec \& Zorn 2015) but never from an ecological point of view.

It is evident from previous studies (Tintner \& Klug 2011; Tintner et al. 2012) on Central European landfills that aboveground vegetation differs visually and measurably from natural or semi-natural vegetation in the surrounding habitat. Plant communities respond to external influences and the importance of site factors to plant species composition has been demonstrated repeatedly (Goslee \& Sanderson 2010). It has been proved that vegetation can indicate characteristics of landfill cover layer and, in some cases (organic waste), can even indicate some characteristics of the underlying waste, including landfill gases (Tintner \& Klug 2011; Tintner et al. 2012). The characteristics of communities growing on landfill surfaces depend on the type of succession, whether it was completely natural or human-induced (Baasch et al. 2012). Hemeroby, urbanity and ruderality are measures of disturbance and human impact that have been used in urban ecology (Hill et al. 2002) and studies on spontaneous succession (Tropek et al. 2010).The diagnostic power of individual species can be estimated by a fidelity measure that compares the presence of species within the community and beyond it. Species with high fidelita values can be treated as diagnostic (Peinado et al. 2013). In addition to species themselves, we can use species indicator values which act as an important tool for evaluating ecological conditions (Ellenberg et al. 1992; Diekmann 2003; Jóźwiak \& Jóźwiak 2014). Even though these values have some limitations, they are widely used in applied plant ecology (Carpenter \& Goodenough 2014). Plant functional traits can also be used; these describe the functional response of vegetation to landuse changes (Garnier et al. 2007). It is also important to estimate how close to nature the vegetation is; human influence on vegetation can be estimated by hemerobic levels (Klotz et al. 2002). So far, no study has been published on human-induced land degradation of dolines with regard to bioindication. The aim of this study is to discover the potential of plant species and plant communities for detecting the locations of waste-filled dolines (doline-dumps) to identify unknown potential pollution sites and degraded habitats. Special diagnostic species that show the presence of doline-dumpsite in the agroecosystems of a polje were identified for individual classes. Since species composition does not entirely describe community functioning, we attempted to find the linkage between floristic composition, structure and ecological conditions of the habitat. In view of the time complexity of doline waste dumps (from recent up to 50-year-old recession plots) and the heterogeneity of dumped waste, we also aim to ascertain whether plant communities can be used as an estimator of the period of time since doline dumping was completed.

\section{METHODS}

\section{GEOGRAPHICAL AND GEOLOGICAL SETTINGS OF THE STUDY AREA}

The study was conducted on Logaško polje, one of the most northern of the 130 poljes in the Dinaric Karst (Gams 1978; Mihevc et al. 2010). Due to intensive afforestation that affected Dinaric Karst in past decades this region is characterized as a landscape coldspot (Perko et al. 2017).

The topography of polje is the result of natural hydro-geomorphic processes and long-term human interventions into the karst geomorphology and hydrogeology. As these combined processes are going on, many dolines have already been overgrown with vegetation, some of them for a couple of years, others even for decades. Apparently they are "masked" by the surrounding agricultural landscape, mainly intensive grasslands (Fig. 1). Previous studies on doline degradation on Logaško polje (Breg 2007) pointed out many potential and existing sites of doline degradation.
Logaško polje is an overflowing ponor polje. A large portion of the polje lies on dolomite, while limestone at the altitude of the plateau represents a 10 to $15 \mathrm{~m}$ higher rock cut terrace. The northern and western parts of the polje consist of early Triassic layered dolomite, with layers from 0.5 to 1 meter thick. Permian deposits of flint sandstone and clay slate appear on the far north edge of Logaško polje. Lower Cretaceous and Cenomanian deposits can be found on the south-east and east part of Logaško polje and consist of a range of grey and dark grey platy limestone, intermitted with grainy bituminous dolomite a few centimetres thick. On the bottom of the polje, located at an altitude between 470 and 480 meters, lie Quaternary river and stream deposits of an average thickness of 3 to 4 meters (Pleničar \& Buser 1970).

Dolines in alluvial (Quaternary deposits) parts of the karst polje are formed by suffusion processes and are differently called by different authors: suffusion dolines (Ford \& Williams 2007), cover dolines (Sauro 2003), 


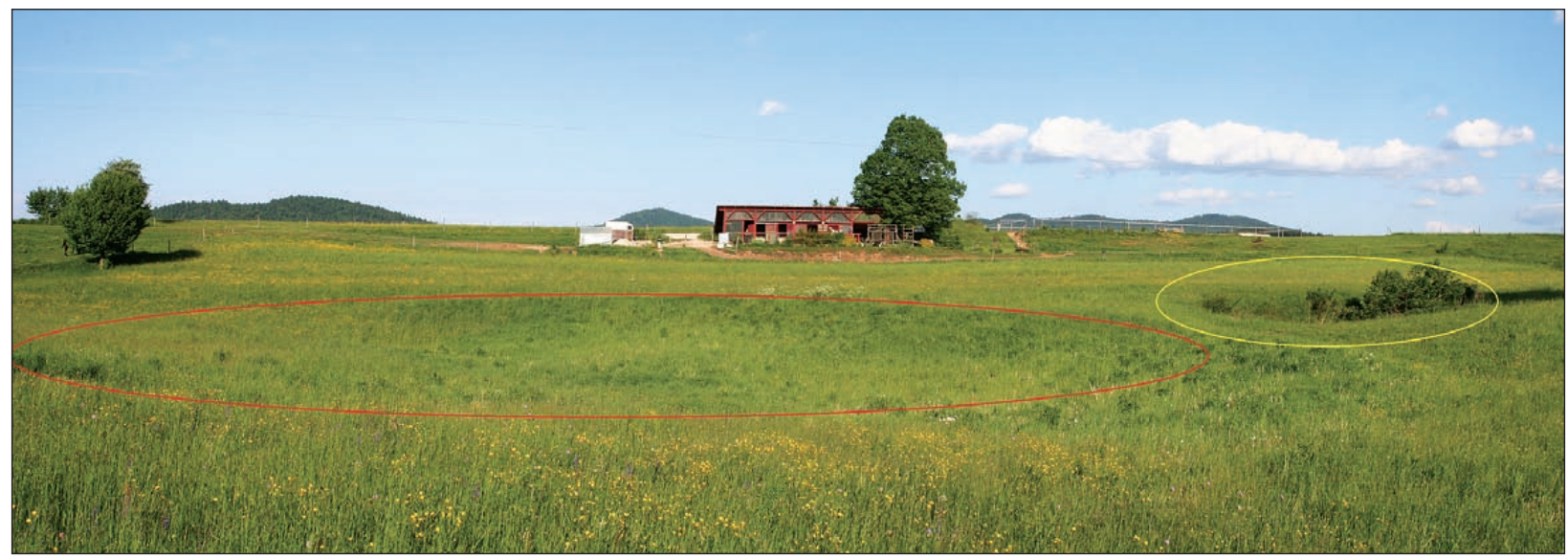

Fig. 1: Approximately 30 years old waste-filled doline (marked with red line), which visually expresses different vegetation (lower grass) from the surrounding grassland and the preserved doline (marked with yellow line).

alluvial dolines (Gams 2003). In the present research the term suffusion doline was selected. The presence of dolines in agro-ecosystems hampers agricultural production; these also appear to be "suitable locations" for waste disposal. They have been mostly backfilled with mixed waste materials of industrial (e.g. organic) or household origin and recently with construction and demolition waste. Nowadays, the study area is a flattish surface covered by grasslands; notwithstanding, some surfaces are recessed due to subsidence of the material that has been used to backfill the dolines (Fig. 1).

\section{HISTORICAL LANDSCAPE ANALYSIS}

Precise locations and shapes of dolines were detected and determined by historical landscape analysis. We used stereo-image processing of historical aerial photographs from 1972 to generate historical 3D digital terrain model and to determine the initial state of doline landform (shape, depth, volume) (Fig. 2). In this study we used and updated the results of study on 3D stereo image processing and electrical resistivity imaging of dolines on Logaško polje published by Breg Valjavec (2014). Detailed explanation of methods and data is available in referenced article. Besides, series of historical aerial photographs $(1975,1986,1989$, 1992, 1994, 1998, 2001, 2006 and 2011) were acquired to determine the period of doline dumping for individual doline (doline-dump age). By visual analysis the landscape history of individual doline and doline-dumpsite was determined and mapped. The map was used as the input data for field study of vegetation while results on the age of individual doline-dumpsite enabled the control data for the age of succession at studied dolines.

\section{ECOLOGICAL EVALUATION}

Ecological evaluation is the basis for all forms of environmental and ecological management, including assess- ment of ecosystem structure and landscape degradation (Burger 2008).

Field surveys were conducted during the optimal season for vegetation sampling (May-June) in order to make a species inventory to determine which plant species had invaded and colonized the doline-dumps. The ecological evaluation started with sampling plots of $5 \times 5 \mathrm{~m}^{2}$, one plot per doline-dump; which consisted of site reconnaissance and phyto-sociological relevés data collection for each plot and estimation of the species coverage according to the Braun-Blanquet approach (Braun-Blanquet 1964). Vegetation data was stored in the Turboveg database management program (Hennekens \& Schaminée 2001).

A data table (164 species X 39 sample sites) was built and was subjected to numerical analysis. For the purpose of numerical analysis, we transformed the cover values into percentages and square rooted. Classification was then carried out by PC-ORD (Mccune \& Mefford 1999), ran in the JUICE 7.0 program (Tichý 2009; Tichý \& Holt 2006). A dendrogram was prepared using Ward's method and Euclidean distance as a measure of resemblance. We used the phi coefficient as a fidelity measure of species to an individual cluster. We also calculated Fischer's exact test and gave a zero fidelity value to species with $\mathrm{P}>0.05$. The threshold phi value (multiplied by 100 in the JUICE program that was used in calculations) set for the species to be considered as characteristic was 40 (Chytrý et al. 2002).

Life forms were determined following Raunkiaer (1934) and Pignatti et al. (2005); ecological indicator values were used after Ellenberg et al. (1992) commonly used to describe substrate conditions (Diekmann 2003) and hemeroby according to Klotz et al. (2002). For interpretation of the ecological conditions, the indicator values were passively projected onto a non-metric multidimensional 


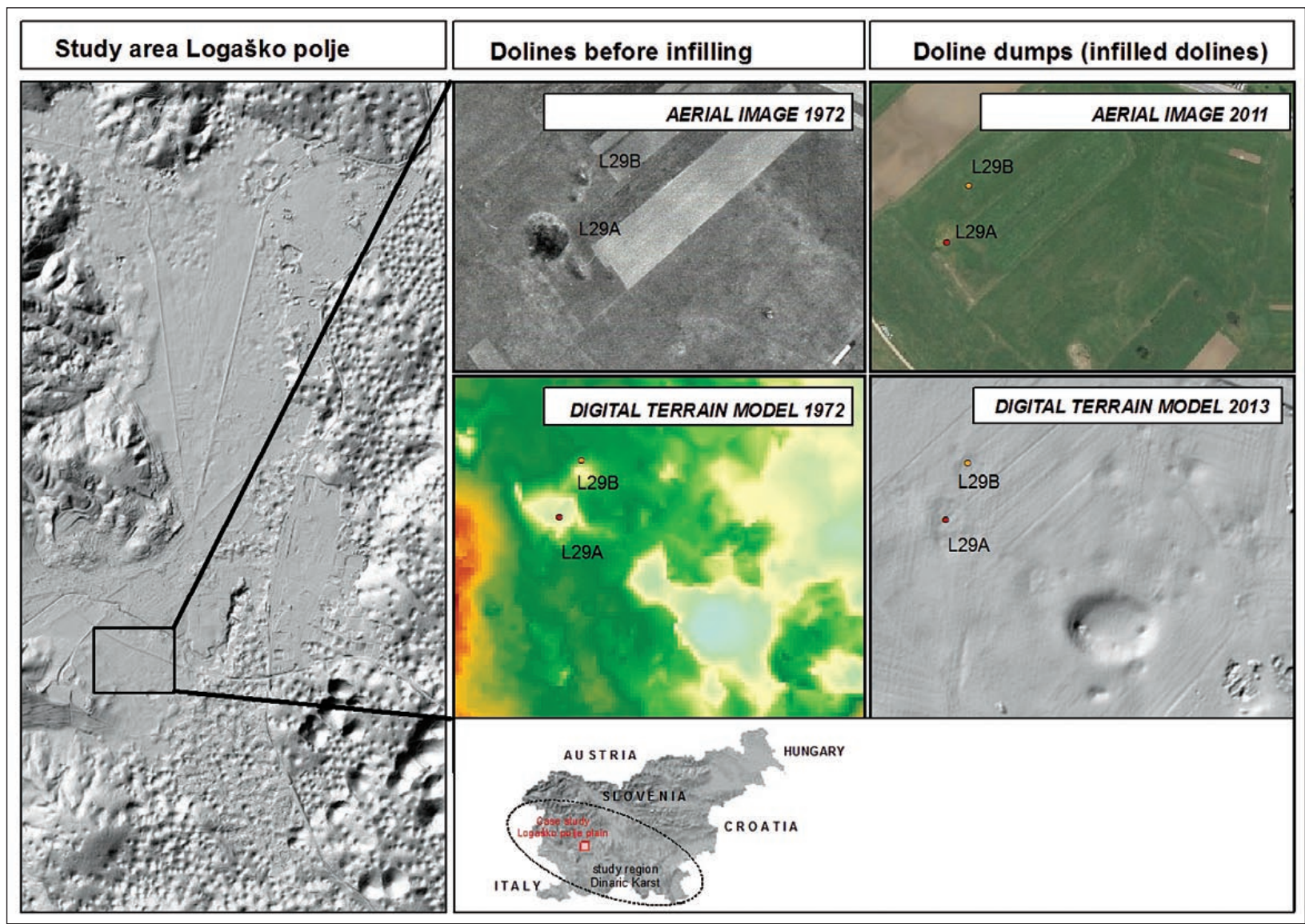

Fig. 2: Study area of Logaško polje (left image) and extended view of two doline-dumps landscape history (L29A, L29B).

scaling (NMDS) diagram in order to reveal ecological relationships among clusters and indicator values; in this way, we could better explain environmental gradients un- derlying the main ordination axes. Differences between clusters were presented by Box-Wishers diagrams in $\mathrm{R}$ project (R Development Core Team 2015).

\section{RESULTS}

CLASSIFICATION OF PLANT COMMUNITIES 30 doline-dumpsites were selected as the sampling sites for further field assessment of plant communities. Landscape history was studied through historical analysis of dolines geomorphology and it shows (Fig. 8, left image) that the most intensive period of doline dumping was in the 1980s and at the beginning of 1990s.

Therefore 19 doline-dumps are $20-30$ years old and six out of 30 doline-dumps are 30-40 years old. Four doline-dumps' vegetation are younger than 10 years and only one doline-dump vegetation is older than 40 years.

Upon preliminary field vegetation study, it can be summarized that doline-dumps inside agricultural grasslands present a circular shape, represented by a lightly concave surface topography of slightly different vegetation, mainly of more eutrophic or ruderal character (see Fig. 1). They cover small surface areas (on average $200 \mathrm{~m}^{2}$ ) and are randomly dispersed in the agricultural landscape of the study area. Their depth (before they were filled) was ranging from 3 to $7 \mathrm{~m}$ on average (which is also the thickness of the dumped material).

With phytocenological assessment of study plots and statistical analysis of Ellenberg indicator values we recognized five clusters that are ecologically well interpretable (Fig. 3, Tab. 2, Fig. 8). The plant communities, sampled at 39 sites in total, were classified into five community clusters $(1,2,3,4$ and 5) corresponding to 


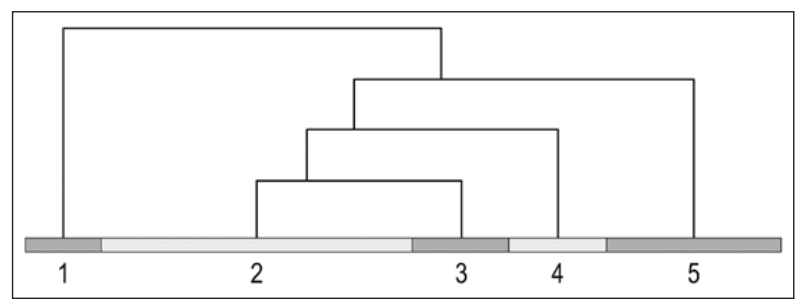

Fig. 3: Dendrogram, Euclidean distance as a resemblance measure and Ward's method. The classification resulted in five clusters: (1) - dry grasslands, (2) - oldest doline-dumps floristically close to mesic grasslands; (3) - mesic grasslands, (4) - annual, eutrophic communities and (5) - perennial, nitrophilous forb communities. two general groups (i and ii) differentiated by absence or presence of human-induced degradation:

(i) Communities of semi-natural grasslands/absence of land degradation: (1) dry grasslands, (3) mesic grasslands; and

(i) Communities of doline-dumps/presence of land degradation: (2) oldest doline-dumps floristically close to mesic grasslands, (4) annual, eutrophic communities and (5) nitrophilous perennial forb communities.

Tab. 1: Synoptic table of five clusters showing the frequency and fidelity of species (upper case).

\begin{tabular}{|c|c|c|c|c|c|c|c|c|c|c|}
\hline Group No. & \multicolumn{2}{|c|}{1} & \multicolumn{2}{|c|}{2} & \multicolumn{2}{|c|}{3} & \multicolumn{2}{|c|}{4} & \multicolumn{2}{|c|}{5} \\
\hline No. of relevés & \multicolumn{2}{|c|}{4} & \multicolumn{2}{|c|}{16} & \multicolumn{2}{|c|}{5} & \multicolumn{2}{|c|}{5} & \multicolumn{2}{|c|}{9} \\
\hline Ranunculus bulbosus & 100 & 96.3 & & --- & . & --- & . & --- & . & --- \\
\hline Carex flacca & 75 & 84.0 & . & --- & . & --- & $\cdot$ & --- & . & --- \\
\hline Carex caryophyllea & 75 & 84.0 & $\cdot$ & $\begin{array}{ll}--- \\
\end{array}$ & $\cdot$ & --- & $\cdot$ & $\begin{array}{ll}--- \\
\end{array}$ & . & -- \\
\hline Bromopsis erecta & 100 & 80.7 & 19 & --- & 20 & --- & $\cdot$ & -- & . & -- \\
\hline Arenaria serpyllifolia & 75 & 79.6 & 6 & --- & . & --- & $\cdot$ & --- & . & --- \\
\hline Festuca rupicola & 75 & 71.4 & $\cdot$ & --- & 20 & --- & $\cdot$ & --- & . & --- \\
\hline Salvia pratensis & 100 & 71.2 & 25 & 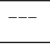 & 20 & --- & 20 & --- & $\cdot$ & -- \\
\hline Vicia lathyroides & 50 & 66.7 & $\cdot$ & --- & $\cdot$ & --- & $\cdot$ & --- & $\cdot$ & --- \\
\hline Galium verum & 50 & 66.7 & . & --- & $\cdot$ & -- & $\cdot$ & --- & $\cdot$ & --- \\
\hline Leucanthemum praecox & 50 & 66.7 & . & --- & $\cdot$ & --- & . & --- & $\cdot$ & --- \\
\hline Sedum annuum & 50 & 66.7 & . & +-- & . & --- & $\cdot$ & --- & $\cdot$ & -- \\
\hline Thlaspi praecox & 50 & 66.7 & . & --- & . & --- & $\cdot$ & --- & . & --- \\
\hline Carex montana & 50 & 66.7 & . & --- & . & --- & $\cdot$ & --- & $\cdot$ & -- \\
\hline Brachypodium pinnatum & 50 & 66.7 & . & --- & $\cdot$ & --- & $\cdot$ & --- & $\cdot$ & -- \\
\hline Leontodon hispidus & 100 & 63.2 & 12 & --- & 60 & --- & 20 & -- & $\cdot$ & --- \\
\hline Trifolium campestre & 50 & 61.3 & 6 & --- & & --- & $\cdot$ & --- & $\cdot$ & --- \\
\hline Medicago lupulina & 100 & 57.0 & 38 & -- & 60 & --- & 20 & -- & $\cdot$ & -- \\
\hline Thymus longicaulis & 50 & 56.7 & 12 & -- & $\cdot$ & --- & $\cdot$ & --- & $\cdot$ & -- \\
\hline Hypericum perforatum & 50 & 51.9 & $\cdot$ & $\begin{array}{ll}--- \\
\end{array}$ & . & $\begin{array}{ll}--- \\
\end{array}$ & 20 & --- & . & -- \\
\hline Briza media & 50 & 45.8 & $\cdot$ & --- & 20 & --- & $\cdot$ & --- & 11 & --- \\
\hline Lotus corniculatus & 75 & 42.3 & 19 & --- & 60 & -- & 20 & -- & • & -- \\
\hline Convolvulus arvensis & $\cdot$ & --- & 75 & 50.3 & 20 & --- & 40 & --- & 11 & -- \\
\hline Trisetum flavescens & 50 & -- & 94 & 35.7 & 60 & --- & $\cdot$ & -- & 89 & -- \\
\hline Arrhenatherum elatius & 75 & --- & 100 & 34.0 & 60 & --- & 40 & --- & 67 & --- \\
\hline Prunella vulgaris & $\cdot$ & --- & 38 & -- & 100 & 70.1 & 20 & --- & 11 & -- \\
\hline Holcus lanatus & $\cdot$ & --- & 62 & --- & 100 & 52.4 & 20 & -- & 56 & -- \\
\hline Daucus carota & 50 & --- & 25 & --- & 80 & 47.2 & 20 & -- & . & -- \\
\hline Cynosurus cristatus & 75 & --- & 56 & --- & 100 & 47.2 & $\cdot$ & --- & 33 & --- \\
\hline Veronica persica & . & -- & 12 & --- & . & --- & 80 & 73.1 & 11 & -- \\
\hline Capsella bursa-pastoris & $\cdot$ & --- & 12 & --- & 20 & --- & 80 & 68.8 & $\cdot$ & -- \\
\hline Lamium purpureum & $\cdot$ & --- & 19 & --- & . & --- & 60 & 60.7 & $\cdot$ & -- \\
\hline Lactuca serriola & $\cdot$ & --- & $\cdot$ & --- & $\cdot$ & --- & 40 & 59.0 & $\cdot$ & --- \\
\hline Chenopodium album & $\cdot$ & --- & $\cdot$ & --- & . & --- & 40 & 59.0 & $\cdot$ & --- \\
\hline Cerastium brachypetalum & $\cdot$ & --- & 6 & --- & $\cdot$ & --- & 40 & 53.1 & . & -- \\
\hline Sonchus asper & $\cdot$ & $\begin{array}{ll}--- \\
\end{array}$ & $\cdot$ & --- & 20 & --- & 40 & 43.1 & $\cdot$ & -- \\
\hline
\end{tabular}




\begin{tabular}{|c|c|c|c|c|c|c|c|c|}
\hline Group No. & 1 & 2 & 3 & & 4 & & 5 & 5 \\
\hline No. of relevés & 4 & 16 & 5 & & 5 & & 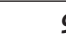 & 9 \\
\hline Plantago major & . --- &.$^{---}$ & $20-$ & $\begin{array}{l}-- \\
\end{array}$ & 40 & 43.1 & . & --- \\
\hline Anthriscus sylvestris &.$^{---}$ & $19^{---}$ & $20^{-}$ & $\begin{array}{c}-- \\
\end{array}$ & . & --- & 89 & \\
\hline Ajuga reptans &.$^{---}$ & $44^{---}$ & $40^{-}$ & $\begin{array}{c}-- \\
\end{array}$ & . & $\begin{array}{c}-- \\
\end{array}$ & 100 & 65.6 \\
\hline Rumex obtusifolius & . --- & $50^{---}$ & $20-$ & $\begin{array}{c}-- \\
-1\end{array}$ & 60 & $\begin{array}{c}-- \\
\end{array}$ & 100 & 54.2 \\
\hline Heracleum sphondylium &.$^{---}$ & $31^{---}$ & $20-$ & $\begin{array}{c}-- \\
\end{array}$ & 20 & $\begin{array}{c}-- \\
\end{array}$ & 78 & 52.4 \\
\hline Alopecurus pratensis & . -- & $12^{---}$ & . - & $\begin{array}{c}-- \\
\end{array}$ & $\cdot$ & --- & 44 & 52.0 \\
\hline Vicia sepium & . -- & $62^{---}$ & $40-$ & --- & 20 & --- & 89 & 47.2 \\
\hline Glechoma hederacea &.-- & $75^{---}$ & $40^{-}$ & $\begin{array}{c}-- \\
\end{array}$ & 20 & $\begin{array}{ll}--- \\
\end{array}$ & 89 & 44.4 \\
\hline Aegopodium podagraria &.$^{---}$ & $6^{---}$ & $\cdot-$ & $\begin{array}{c}-- \\
\end{array}$ & 20 & 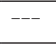 & 44 & 43.5 \\
\hline Poa trivialis &.$^{---}$ & $69^{---}$ & $80-$ & $\begin{array}{c}-- \\
\end{array}$ & 60 & --- & 100 & 39.4 \\
\hline Colchicum autumnale & . --- & . --- & $20-$ & --- & . & --- & 33 & 36.7 \\
\hline Dactylis glomerata & $100^{---}$ & $100^{---}$ & 100 & $\begin{array}{c}-- \\
\end{array}$ & 60 & --- & 100 & --- \\
\hline Leucanthemum vulgare & $100^{---}$ & $88^{---}$ & $100^{-}$ & $\begin{array}{c}-- \\
\end{array}$ & 60 & --- & 100 & $\begin{array}{c}-- \\
\end{array}$ \\
\hline Taraxacum officinale & $25^{---}$ & $94^{---}$ & $100^{-}$ & --- & 100 & --- & 100 & --- \\
\hline Plantago lanceolata & $25^{---}$ & $100^{---}$ & $100^{-}$ & --- & 80 & --- & 89 & --- \\
\hline Achillea millefolium & $75^{---}$ & $100^{23.3}$ & $100^{-}$ & $\begin{array}{c}-- \\
\end{array}$ & 80 & --- & 56 & --- \\
\hline Trifolium pratense & $75^{---}$ & $8^{---}$ & $100^{-}$ & --- & 80 & -- & 44 & -- \\
\hline Veronica chamaedrys &.$^{---}$ & $81^{---}$ & $100^{-}$ & --- & 60 & --- & 100 & --- \\
\hline Cerastium holosteoides & $50^{---}$ & $88^{---}$ & $100^{-}$ & $\begin{array}{c}-- \\
\end{array}$ & 80 & --- & 33 & --- \\
\hline Ranunculus acris & $25^{---}$ & $88^{---}$ & $100^{-}$ & --- & 20 & --- & 78 & --- \\
\hline Knautia arvensis & $75^{---}$ & $69^{---}$ & $100^{-}$ & $\begin{array}{c}-- \\
\end{array}$ & 40 & $\begin{array}{ll}--- \\
\end{array}$ & 67 & --- \\
\hline Centaurea carniolica & $50^{---}$ & $69^{---}$ & $100^{-}$ & $\begin{array}{c}-- \\
\end{array}$ & 40 & $\begin{array}{ll}-- \\
\end{array}$ & 67 & --- \\
\hline Galium mollugo & $25^{---}$ & $81^{---}$ & $40-$ & $\begin{array}{c}-- \\
\end{array}$ & 40 & --- & 89 & --- \\
\hline Centaurea jacea & $50^{---}$ & $69^{---}$ & 100 & --- & 20 & --- & 67 & $\overline{---}$ \\
\hline Festuca pratensis & $25^{---}$ & $69^{---}$ & 100 & $\begin{array}{c}-- \\
\end{array}$ & 40 & $\begin{array}{l}-- \\
\end{array}$ & 67 & -- \\
\hline Crepis biennis & .--- & $69^{---}$ & $80^{-}$ & $\begin{array}{c}-- \\
\end{array}$ & 40 & $\begin{array}{ll}--- \\
\end{array}$ & 89 & --- \\
\hline Rumex acetosa & $25^{---}$ & $56^{---}$ & $100^{-}$ & --- & 20 & --- & 89 & --- \\
\hline Poa pratensis & $75^{---}$ & $62^{---}$ & $80^{-}$ & --- & 20 & --- & 56 & --- \\
\hline Allium carinatum & $50^{---}$ & $69^{---}$ & $80^{-}$ & $\begin{array}{c}--- \\
\end{array}$ & 20 & $\begin{array}{ll}--- \\
\end{array}$ & 56 & --- \\
\hline Veronica arvensis & $50^{---}$ & $75^{---}$ & $40-$ & $\begin{array}{c}-- \\
\end{array}$ & 80 & $\begin{array}{ll}--- \\
\end{array}$ & 22 & --- \\
\hline
\end{tabular}

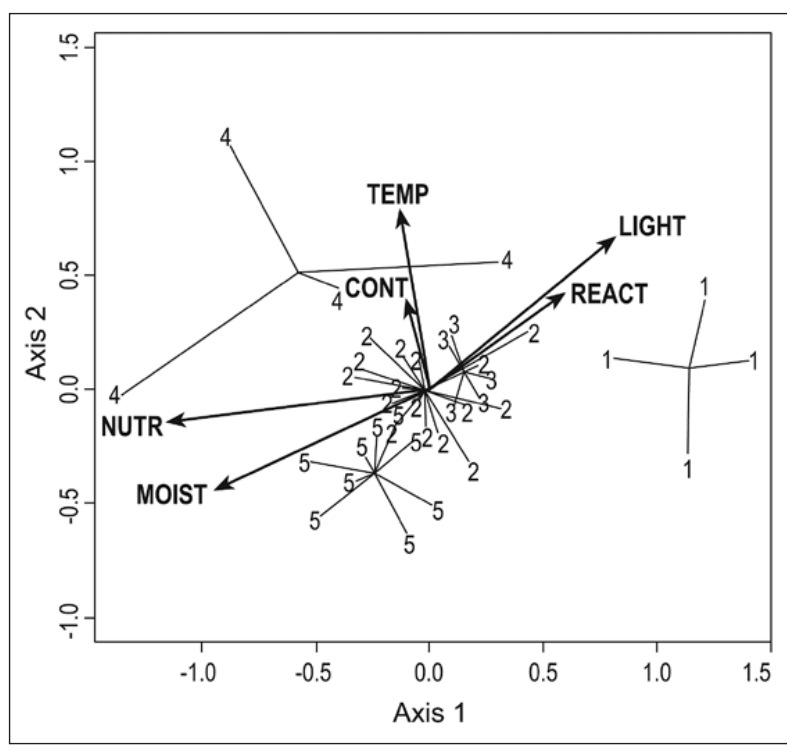

Fig. 4: NMDS ordination diagram with passively projected Ellenberg bioindicator values. Detailed legend is specified in Fig. 3.

\section{COMMUNITIES OF SEMI-NATURAL GRASSLANDS}

Dry grassland communities (cluster 1) are the characteristic vegetation of dry shallow rendzic laptosols on carbonate bedrock. They appear on the edge of polje where limestone bedrock is exposed and it continues to the karst plateau. Some characteristic species of this group are $R a$ nunculus bulbosus, Bromus erectus and Carex caryophyllea (Tab. 1/1). Considering Ellenberg indicator values, this vegetation type appears on the driest sites with high $\mathrm{pH}$ (alkaline), nutrient-poor soils and highly exposed to light (Fig. 5). These conditions enable the establishment of a relatively high proportion of therophytes (Fig. 3). Among all studied vegetation are dry grasslands the most natural ones (Fig. 4) since the proportion of oligohemerobic and mesohemerobic species is the highest.

Mesic grasslands (cluster 3) are another grassland diagnostic community developed on humid deep loamy soils of polje (Fig. 3). The vegetation in these grasslands is very similar to vegetation of old doline-dumps and 
Tab. 2: The results of historical landscape study and ecology study.

\begin{tabular}{|c|c|c|c|c|}
\hline \multirow[b]{2}{*}{ DOLINE } & \multicolumn{2}{|c|}{ HISTORICAL LANDSCAPE STUDY } & \multicolumn{2}{|l|}{ ECOLOGY STUDY } \\
\hline & TYPE OF SITE & $\begin{array}{c}\text { AGE OF } \\
\text { SUCCESSION } \\
\text { (period since doline } \\
\text { backfilling was } \\
\text { completed) }\end{array}$ & $\begin{array}{c}\text { NUMERICAL } \\
\text { VEGETATION ANALYSIS } \\
\text { (field survey \& dendrogram) }\end{array}$ & $\begin{array}{l}\text { CLASSIFICATION OF } \\
\text { COMMUNITIES }\end{array}$ \\
\hline $\mathrm{T}^{\mathrm{b}}$ & grassland & / & 1 - dry grasslands & \multirow{4}{*}{$\begin{array}{l}\text { (i) } \\
\text { Communities of } \\
\text { semi-natural } \\
\text { grasslands } \\
\text { absence of land } \\
\text { degradation }\end{array}$} \\
\hline $\mathrm{T} 2^{\mathrm{b}}$ & grassland & / & 1 - dry grasslands & \\
\hline $\mathrm{T}^{\mathrm{b}}$ & grassland & / & 1 - dry grasslands & \\
\hline $\mathrm{T}^{\mathrm{b}}$ & grassland & / & 1 - dry grasslands & \\
\hline L6 & backfilled doline & 26 & 2 - oldest doline-dumps & \multirow{16}{*}{$\begin{array}{l}\text { (ii) } \\
\text { communities of } \\
\text { doline-dumps } \\
\text { presence of land } \\
\text { degradation }\end{array}$} \\
\hline $\mathrm{L} 2$ & backfilled doline & 29 & 2 - oldest doline-dumps & \\
\hline L13 & backfilled doline & 40 & 2 - oldest doline-dumps & \\
\hline L4 & backfilled doline & 29 & 2 - oldest doline-dumps & \\
\hline L14 & backfilled doline & 40 & 2 - oldest doline-dumps & \\
\hline T4 a,b & grassland & 1 & 2 - oldest doline-dumps & \\
\hline L29B & backfilled doline & 26 & 2 - oldest doline-dumps & \\
\hline $\mathrm{L} 24$ & backfilled doline & 23 & 2 - oldest doline-dumps & \\
\hline L17 & backfilled doline & 26 & 2 - oldest doline-dumps & \\
\hline $\mathrm{L} 5$ & backfilled doline & 26 & 2 - oldest doline-dumps & \\
\hline L28 & backfilled doline & 29 & 2 - oldest doline-dumps & \\
\hline L1 & backfilled doline & 40 & 2 - oldest doline-dumps & \\
\hline L11 & backfilled doline & 35 & 2 - oldest doline-dumps & \\
\hline L12A & backfilled doline & 40 & 2 - oldest doline-dumps & \\
\hline L15 & backfilled doline & 43 & 2 - oldest doline-dumps & \\
\hline L21 & backfilled doline & 23 & 2 - oldest doline-dumps & \\
\hline $\mathrm{T}^{\mathrm{b}}$ & grassland & I & 3 - mesic grasslands & \multirow{5}{*}{$\begin{array}{l}\text { (i) } \\
\text { Communities of } \\
\text { semi-natural } \\
\text { grasslands / } \\
\text { absence of land } \\
\text { degradation }\end{array}$} \\
\hline$T 7^{b}$ & grassland & I & 3 - mesic grasslands & \\
\hline $\mathrm{T} 1^{\mathrm{b}}$ & grassland & / & 3 - mesic grasslands & \\
\hline $\mathrm{T}^{\mathrm{b}}$ & grassland & / & 3 - mesic grasslands & \\
\hline L16 $^{\mathrm{a}}$ & backfilled doline & 29 & 3 - mesic grasslands & \\
\hline L29A & backfilled doline & 9 & 4 - annual, eutrophic communities & \multirow{5}{*}{$\begin{array}{l}\text { (ii) } \\
\text { communities of } \\
\text { doline-dumps } \\
\text { / } \\
\text { presence of land } \\
\text { degradation }\end{array}$} \\
\hline L3 & backfilled doline & 9 & 4 - annual, eutrophic communities & \\
\hline LA1 & backfilled doline & 9 & 4 - annual, eutrophic communities & \\
\hline LA2 & backfilled doline & 9 & 4 - annual, eutrophic communities & \\
\hline L12B & backfilled doline & 40 & 4 - annual, eutrophic communities & \\
\hline $\mathrm{L} 25$ & backfilled doline & 23 & 5 - nitrophilous perennial forb communities & \multirow{9}{*}{$\begin{array}{l}\text { (ii) } \\
\text { communities of } \\
\text { doline-dumps } \\
\text { presence of land } \\
\text { degradation }\end{array}$} \\
\hline L26 & backfilled doline & 23 & 5 - nitrophilous perennial forb communities & \\
\hline L27 & backfilled doline & 23 & 5 - nitrophilous perennial forb communities & \\
\hline L22 & backfilled doline & 23 & 5 - nitrophilous perennial forb communities & \\
\hline L23 & backfilled doline & 23 & 5 - nitrophilous perennial forb communities & \\
\hline $\mathrm{L} 7$ & backfilled doline & 26 & 5 - nitrophilous perennial forb communities & \\
\hline L8 & backfilled doline & 26 & 5 - nitrophilous perennial forb communities & \\
\hline L9 & backfilled doline & 26 & 5 - nitrophilous perennial forb communities & \\
\hline L18 & backfilled doline & 29 & 5 - nitrophilous perennial forb communities & \\
\hline
\end{tabular}

a sits that were classified different by individual method.

${ }^{\mathrm{b}}$ reference sites that don't have data on age of succession they are considered to be grasslands. 

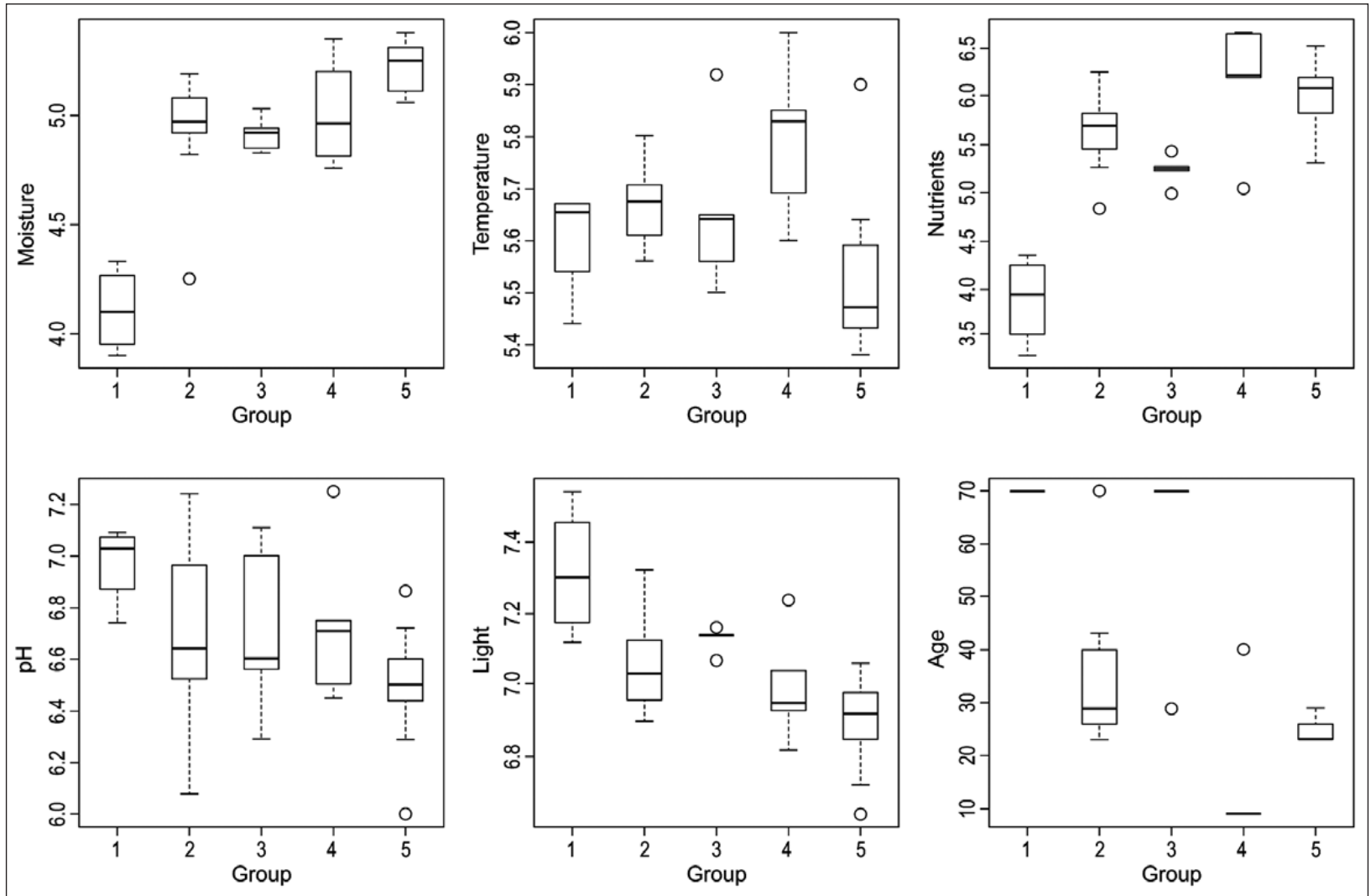

Fig. 5: Comparison of ecological conditions in plant communities estimated by Ellenberg bioindicator values.
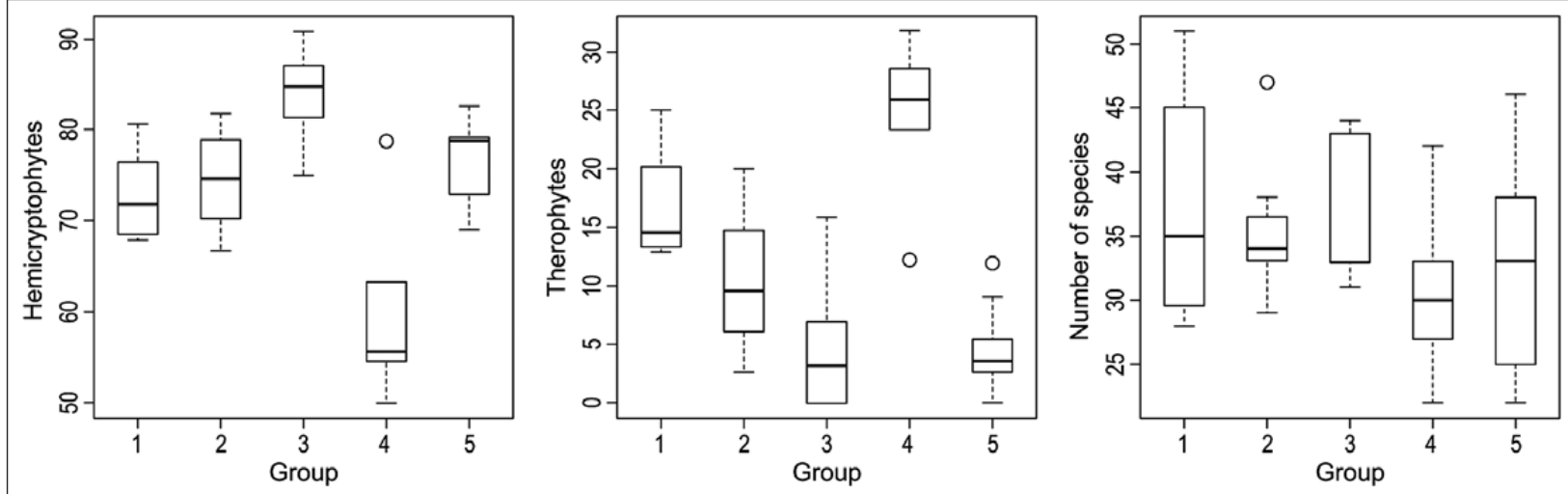

Fig. 6: Comparison of life history traits (only hemicryptophytes and therophytes are presented) and species richness. The median, $25 \%-75 \%$ of values, non-outlier range and outliers are presented here.

some plots mediate between the two groups (Tab. 1). Diagnostic species of this group are Prunella vulgaris, Holcus lanatus, Daucus carota and Cynosurus cristatus (Tab. 1/3). These sites show an intermediate site condition among all identified clusters. In comparison to the most similar old doline-dumps, these communities are poorer in nutrients and more light-demanding (Fig. 5). These communities form a close canopy of hemicryptophytes, preventing therophytic species to settle (Fig. 6). Compared to old doline-dumps, this type of vegetation is more natural, since more oligohemerobic and mesohemerobic species are present and fewer euhemerobic and polyhemerobic ones (Fig. 7).

Communities of semi natural grasslands are considered as referential sites where no degradation occurred.

\section{COMMUNITIES OF DOLINE-DUMPS}

Oldest doline-dumps (cluster 2) are floristically close to mesic grasslands. These dolines were backfilled a long time ago, on average 29 years. Agro-meliorations took 


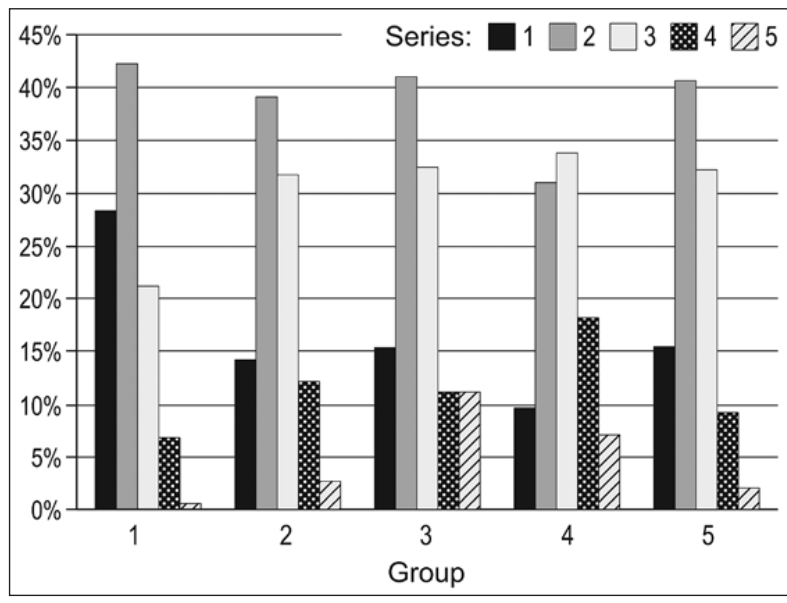

Fig. 7: Comparison of hemeroby between five groups. Legende: series 1 - oligohemerob, 2 - mesohemerob, 3 - $\beta$ euhemerob, $4-\alpha$ euhemerob, 5 - p polyhemerob, for groups see Fig. 3.

place in Logaško polje in late 1980s to improve agricultural land use. Dolines that were backfilled during agromeliorations and before 1990 were covered with a fertile topsoil layer and these surfaces were further used as croplands and grasslands. The diagnostic species of this group are Convolvulus arvensis, Trisetum flavescens and Arrhentherum elatius (Tab. 1/2). These stands show high similarity to the mesic grasslands of the cluster 3 .

Middle-aged doline-dumps dominated by nitrophilous perennial forb communities (cluster 5) are diagnostic for dolines that were filled around 20 years ago, on average of 23 years (Fig. 3). The diagnostic species, with highest abundance, of this group are Anthriscus sylvestris, Ajuga reptans, Rumex obtusifolius, Alopecurus pratensis, Heracleum sphondyllium, Vicia sepium, Glechoma hederacea, Aegopodium podagraria, Poa trivialis and Colchicum autumnale (Tab.1/5). The communities are humid and nutrient-rich (Fig. 5). The proportion of hemicriptophytics is high and there is a relatively low proportion of therophytic species (Fig. 6). These communities are dominated by perennial and ruderal forb species.

Annual, eutrophic communities (cluster 4) (Fig. 3), represent the youngest doline-dumps, covered by annual, eutrophic communities as an evidence of recent land disturbance. Considering results of historical landscape analysis, these dolines have been recently filled, the median is 9 years. Considering socio-economic

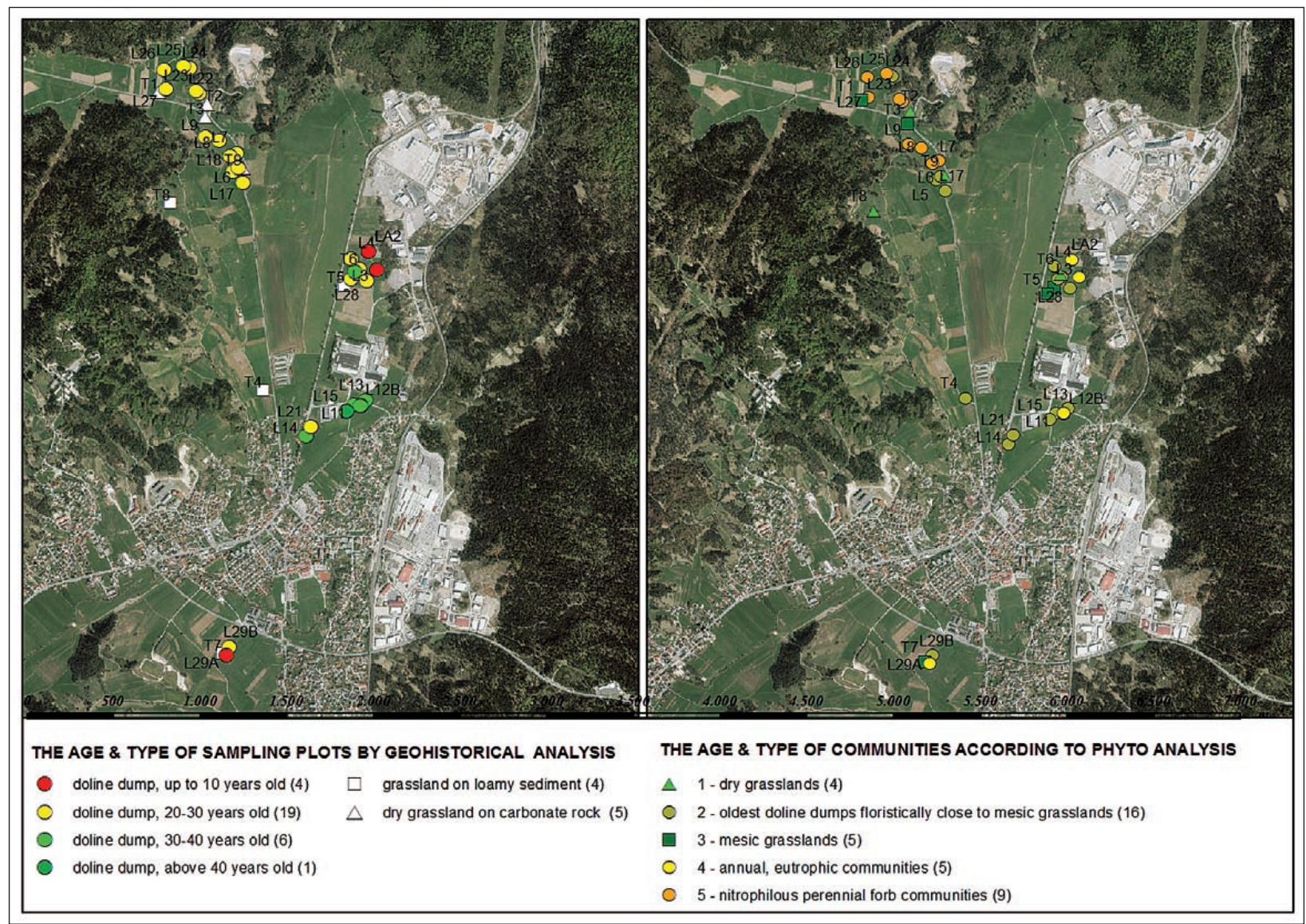

Fig. 8: Comparison of results of historical analysis and results of ecological evaluation. 
development the intensity of agricultural production on Logaško polje decreased at the beginning of millennium, due to the increase of urbanization. Therefore there was less need for arable land and the waste dumped in dolines was covered with infertile top layers usually consisting of construction and demolition waste or even remained uncovered until the aboveground vegetation developed, naturally or human-induced. The prevailing land use is extensive meadows. During extensive field work in the spring, we noted that grasslands are fertilized either by organic or/and mineral fertilizers to improve the ecological (nutrition) conditions of cover layer. This can also be evident by the presence of eutrophic communities and species at these sites. The diagnostic species of the group are Capsella-bursa pastoris, Veronica persica and Lamium purpureum (Tab. 1/4). The sites are nutrient-rich and warm (Fig. 5). The proportion of theropytic species is high due to open stands (Fig. 6). These stands are the least natural of all, being dominated by euhemerobic species (Fig. 7). In these communities, weed species are well represented, showing the initial, eutrophic character of the communities.

Relations among clusters can be seen on an ordination diagram (Fig. 4) and show that dry grasslands appear with the highest light and reaction, showing a relatively distant position in the diagram. The highest temperature and continentality (well pronounced extreme conditions) are in stands of annual, eutrophic communities, while the other three groups form a fairly homogenous group, from perennial nitrophilous forb communities to mesic grasslands. The highest trophic status includes annual, eutrophic (youngest doline dump) and nitrophilous forb communities (middle-aged doline dump) that are at the beginning of the successional sere. Recent waste and municipal solid waste slag result in a ruderal pioneer stage dominated by weedy species of open soil. Ways of further succession from this stage cannot yet be predicted with certainty as also Tintner and Klug (2011) claimed.

In the figure 6 , the median, $25 \%-75 \%$ of values, non-outlier range and outliers are presented. Detailed legend is specified in Fig. 3.

It is also important to estimate how close to nature the vegetation is; human influence on vegetation was estimated by hemerobic levels (Klotz et al. 2002). The comparison of hemeroby between all five groups (Fig. 7) shows that series 1 (dry grassland) correspond to oligohemerob, series 2 to mesohemerob (oldest dolinedumps), series 3 (mesic grassland) to euhemerob, series 4 (annual, eutrophic communities) to euhemerob and series 5 (perennial, nitrophilous forb communities) to polyhemerob.

\section{DISCUSSION}

\section{GENERAL DISCUSSION ON COMMUNITIES OF DEGRADED DOLINES}

Degraded dolines can be detected by sampling vegetation (plants species and plant communities) within cultivated and natural grasslands of agricultural landscapes. According to Gutiérrez et al. (2014) the illegal waste dumping on Logaško polje has affected changes in karst landforms. Besides, backfilling of dolines indirectly influences on the extinction of rare species that are common for dolines as ecological (climate) refugia (Batori et al. 2014). The aboveground vegetation of waste-filled dolines differs visually and measurably from natural or semi-natural vegetation in the surrounding agricultural (dry and mesic) grasslands and thus influences grassland biodiversity in total.

The time and cost-demanding assessment by physical or chemical parameters could be simplified by the vegetation ecology approach as Tintner and Klug (2011) defended. Plant communities of waste-filled dolines do respond to external influences and the importance of site factors to plant species composition has been demon- strated in accordance to referenced literature (Goslee \& Sanderson 2010).

We succeeded in confirming that indicator species of cluster 1 (dry grasslands) were sampled on carbonate bedrock (limestone, dolomite), where extensive grasslands prevail due to shallow soils over carbonate bedrock. These species show the highest level of naturalness and a low level of hemeroby (Hill et al. 2002). In these conditions, the reaction is high, the availability of nutrients is low and there is fairly high species richness (Merunková \& Chytrý 2012). Hemicriptophytes dominate in this type of vegetation, although sporadically we can also find some therophytes (Batalha et al. 2015). Dry grasslands vegetation is a typical landscape feature of the karst landscape (Kaligarič \& Ivajnšič 2014). By means of our analysis, we could identify this vegetation type in the field and distinguish the natural vegetation of dry grasslands from the vegetation on doline-dumps. This vegetation can be classified within the class of grasslands on base-rich soils Festuco-Brometea (Pipenbaher et al. 2011). 
The second and third clusters can be treated as cultivated (fertilized) mesic grasslands (meadows) of alluvial plain and the oldest doline-dumps, floristically close to mesic grassland. This type of vegetation can develop either over deeper, fertile soils, formed on loamy unconsolidated Holocene river sediments or over dolinedumps with a thicker cover layer of allochthonous fertile soil. The sites are humid and have a neutral reaction. Hemicryptophytic species are here dominant, forming dense stands and not allowing therophytes to establish (Čámská \& Skálová 2012; Šilc et al. 2014).

The fourth cluster is dominated by annual, eutrophic communities. These communities are characteristic of disturbed surfaces, since disturbance favours annual and ruderal species. We found communities with the highest hemeroby within this cluster. The sites are nutrient rich, humid, with relatively high reaction (Perronne et al. 2014; Sans et al. 2014). Since these sites are warm and have a relatively high reaction, we presume that there is carbonate gravel and rock in the upper horizons of the backfilling material. These habitats have high potential to be settled by invasive species (such as Parthenocissus or Erigeron), so management of these sites must be done with prudence (Zisenis 2015). These communities indicate that the dolines have been covered with gravel (probably from construction waste). The relatively early successional stage, marked by the therophytic communities (Sojneková \& Chytrý 2015), indicates that the backfilling has been done recently. No reference site is included in this cluster, only dumped dolines (5 of them), predominantly younger doline-dumps that were filled up to 10 years ago. This vegetation can be classified within the class of annual weed communities Stellarieatea mediae (Šilc \& Čarni 2007).

The fifth cluster represents nitrophilous forb communities. These communities settle on sites with high nutrient availability. The species are mainly hemicryptophytic, often spread by rhizomes and build dense stands. The sites are humid and fertile (Pastor et al. 2014; Shushpannikova 2014). These communities indicate that at least the upper horizons of the doline have been filled with organic waste, which has provided the fertility to the site. Doline-dumps (9 of them) included in this cluster are predominantly dolines that were filled on average 25 years ago. These communities are classified within the class of tall-herb semi-natural forb vegetation of nutrient rich sites Galio-Urticetea (Čarni 1994).

\section{THE SUCCESSION SERES}

In accordance to Baasch et al. (2012) results the structure of communities depends on the age of succession which is diverse from communities at undisturbed dry and mesic grasslands. The revegetation and succession time of doline-dumps is a very important variable that influences the stability of a meadow species community. We noted that after an extended succession period (20-40 years), species communities on doline-dumps became very similar to cultivated meadows and it was more difficult to differentiate them from the surrounding grasslands. This corresponds to the results obtained by Prach and Pýšek (2001), who mentioned that in smaller disturbed sites, succession towards natural vegetation is easier and more direct. In this case, we needed to use for identification of doline-dumps, indicator plant species from the group of nitrophilous forb communities, which provides linkage with previous successional phases (e.g. Urtica doica, Rumex obtusifolia etc.). The results show that upper layers consist of relatively fertile soil, although we are not quite sure about the lower layers. This vegetation can be classified as Molinio-Arrhenatheretea (Šilc et al. 2014), a class of anthropogenic pastures and meadows on deeper, fertile soils.

The results confirm that dry grasslands are beyond the successional sere. The succession begins with annual, synanthropic vegetation, develops towards perennial nitrophilous forb communities and succession is arrested with mesic grasslands.

Annual and eutrophic vegetation appears on new doline-dumps but this type of vegetation can be replaced by perennial species in a short period of time (Prach 1987, Sojneková \& Chytrý 2015). In comparison with abandoned fields (non-cultivated), where this change appears in a few years, in our case it is a relatively long process, lasting up to 10 years, due to the type of top cover material, presumably construction and demolition waste or excavated materials.

In the study area, the next successional stage consists of nitrophilous perennial forb species that dominate the sites for the next period of 10 years. These mid-successional species do not allow the further development of the succession sere (Bartha et al. 2014). Mowing and exporting material (nutrients) from the ecosystem (Herbst et al. 2013) has enabled the development of communities with lower nutrient demand.

The next successional stage is represented by the oldest doline-dumps, which are similar to mesic grasslands. This stage is dominated by Arrheatherum elatius, a competitive nitrophilous grass. This species loses its competitive power in some stands in this and further successional stages, probably due to the lack of phosphorous in the soil (Prach et al. 2007). The oldest doline-dumps are floristically very close to mesic grassland and the transition is sometimes difficult to detect, especially in cases in which mesic meadows are fertilized (Gustavsson et al. 2007). According to Prach et al. (2014), the succession in Central Europe would reach semi-natural vegetation in 
about 25 years, with the species composition changing a little later on. Our study showed similar results, as after 30 years the vegetation of the oldest doline-dumps very much resembles mesic grasslands. In well-established, mown communities, the trophic status became lower. According to Tintner and Klug (2011) sandy to loamy material with thick cover layers that is characteristic for oldest doline-dumps (covered with thick- loamy soils during agro-meliorations) vegetation develops very close to community of mesic meadows with high successional stability and the classification of these two communities is possible only by diagnostic plants. It was reported by Prach et al. (2013) that invasive species sometimes interfere in the succession line; however, our results showed that such species do not play an important role in the succession sere. We can confirm that spontaneous succession is suitable for the creation of mesic grasslands on waste-filled dolines.

\section{CONCLUSION}

The geomorphological analysis and historical records were the basis for the vegetation assessment which reveals the spatio-temporal patterns and provides the explanation for ecological processes occurring at the individual doline-dump. At Logaško polje hydro-geomorphic processes are continuously forming new suffusion dolines and reshaping existing ones. Doline landform in combination with thermal inversion enables landforminduced-vegetation which is destroyed by doline backfilling. Eventually, a unique karst landform and its role of habitat (refugia) disappeared for ever. However, vegetation patterns of such degraded grasslands differ from the patterns of surrounding non-degraded grasslands. Thus, we conclude that vegetation is a reliable bio-indicator for the detection of doline-dumps in agricultural grassland landscapes. The revegetation and succession time of do- line-dumps is a very important variable that influences the stability of meadow species community. The natural succession of doline-dump begins with annual, synanthropic vegetation, develops towards perennial nitrophilous forb communities and stops at mesic grasslands that are maintained by regular mowing. We noted that after an extended succession period (20-40 years), species communities on doline-dumps became very similar to cultivated meadows and thus it's more difficult to differentiate them from the surrounding grasslands.

We were able to assess unsustainable landscape management practices, such as inappropriate waste disposal, although the methods used should be further tested in comparable vulnerable agricultural landscapes and other doline types (e.g. solution dolines) in environments with different geological and ecological conditions.

\section{ACKNOWLEDGMENTS}

The study was financially supported by the Ministry of Higher Education, Science and Technology of the Republic of Slovenia (OP.13.2.1.01.0067) and the Slovenian Research Agency (P1-0236 to AČ).
We would like to thank Iztok Sajko for the preparation of some graphical material.

\section{REFERENCES}

Baasch, A., Kirmer, A. \& S. Tischew, 2012: Nine years of vegetation development in a post mining site: effects of spontaneous and assisted site recovery.- Journal of Applied Ecology, 49, 251-260, Oxford. DOI: http://dx.doi.org/10.1111/j.1365-2664.2011.02086.x
Baroni, C., Armiraglio, S., Gentili, R. \& A. Carton, 2007: Landform-vegetation units for investigating the dynamics and geomorphologic evolution of alpine composite debris cones (Valle dell'Avio, Adamello Group, Italy).- Geomorphology, 84, 59-79. DOI: http://dx.doi.org/10.1016/j.geomorph.2006.07.002 
Bartha, S., Szentes, S., Horváth, A., Házi, J., Zimmermann, Z., Molnár, C., Dancza, I., Margóczi, K., Pál, R.W., Purger, D., Schmidt, D., Óvári, M., Komoly, C., Sutyinski, Z., Szabó, G., Csathó, A., Juhász, M., Penksza, K. \& Z. Molnár, 2014: Impact of mid-successional dominant species on the diversity and progress of succession in regenerating temperate grasslands.Applied vegetation science, 17, 2, 201-213.

Batalha, M.A., Pipenbaher, N., Bakan, B., Kaligarič,M. \& S. Škornik, 2015: Assessing community assembly along a successional gradient in the North Adriatic Karst with functional and phylogenetic distances.Oecologia, 178, 4, 1205-1214.

Bátori, Z., Csiky, J., Erdős, L., Morschhauser, T., Torok, P. \& L. Kormoczi, 2009: Vegetation of the dolines in Mecsek mountains (south Hungary) in relation to the local plant communities.- Acta Carsologica, 38, 2-3, 237-252. DOI: http://dx.doi.org/10.3986/ ac.v38i2-3.125

Bátori, Z., Farkas, T., Erdös, L., Tölgyesi, C., Körmöczi L. \& A. Vojtkó, 2014: A comparison of the vegetation of forested and non-forested solution dolines in Hungary: a preliminary study.- Biologia, 69, 10: 1339-1348.

Braun-Blanquet, J., 1964: Pflanzensoziologie. Grudzüge der Vegetationskunde. 3. Auflage. Springer Verlag, pp. 865 , Wien.

Breg, M., 2007: Degradation of dolines on Logaško polje (Slovenia). Acta Carsologica, 36, 2, 223-231. DOI: http://dx.doi.org/10.3986/ac.v36i2.191

Breg Valjavec, M., 2014: Study of filled dolines by using 3D stereo image processing and electrical resistivity imaging.- International Journal of Speleology, 43, 1, 57-68. DOI: http://dx.doi.org/10.5038/1827-806X.43.1.6

Breg, M. \& M. Zorn, 2015: Degraded karst relief: wastefilled dolines.- In: Daniels, J.A.: Advances in environmental research, 40, Nova Science Publishers, pp. 77-95. New York.

Burger, J., 2008: Environmental management: Integrating ecological evaluation, remediation, restoration, natural resource damage assessment and long-term stewardship on contaminated lands.- Science of the Total Environment, 400, 6-19. DOI: http://dx.doi. org/10.1016/j.scitotenv.2008.06.041

Čámská, K. \& H. Skálová, 2012: Effect of low-dose N application and early mowing on plant species composition of mesophilous meadow grassland (Arrhenatherion) in Central Europe.- Grass and Forage Science, 67, 3, 403-410.

Čarni, A., 1994: Les associations des ourlet nitrophiles dans le sud-est de la Slovénie comme indicateur des habitats.- Colloques phytosociologiques, 22, 467-497.
Carpenter, W. \& A. Goodenough, 2014: How robust are community-based plant bioindicators? Empirical testing of the relationship between Ellenberg values and direct environmental measures in woodland communities.- Community Ecology, 15, 1, 1-11. DOI: http://dx.doi.org/ 10.1556/ComEc.15.2014.1. 1

Cernatič Gregorič, A. \& M. Zega, 2010: The impact of human activities on dolines (sinkholes) - typical geomorphologic features on Karst (Slovenia) and possibilities of their preservation.- Geographica Pannonica, 14, 4, 109-117.

Chytrý, M., Tichý, L., Holt, J. \& Z. Botta-Dukát, 2002: Determination of diagnostic species with statistical fidelity measures.- Journal of Vegetation Science, 13, 79-90. DOI: http://dx.doi.org/10.1111/j.16541103.2002.tb02025.x

Diekmann, M., 2003: Species indicator values as an important tool in applied plant ecology - a review.Basic and Applied Ecology, 4, 493-506. DOI: http:// dx.doi.org/10.1078/1439-1791-00185

Ellenberg, H., Weber, H.E., Düll, R., Wirth, V., Werner, W. \& D. Paulißen, 1992: Zeigerwerte von Pflanzen in Mitteleuropa.- Scripta Geobotanica 18, 1-248.

Fišer, C., Pipan, T. \& D.C. Culver, 2014: The vertical extent of groundwater metazoans: an ecological and evolutionary perspective.- Bioscience, 64, 11, 971979. DOI: http://dx.doi.org/ 10.1093/biosci/biu148

Ford, D.C. \& P.W. Williams, 1989: Karst Geomorphology and Hydrology.- Academic Division of Unwin Hyman Ltd, pp. 601, London.

Ford, D. \& P.W. Williams, 2007: Karst hydrogeology and geomorphology. John Whiley \& Sons, pp. 562, Chichester.

Gams, I., 1978: The polje: the problem of definition.Zeitschrift für Geomorphologie, 22, 2, 170-181.

Gams, I., 2000: Doline morphogenetical processes from global and local viewpoints.- Acta Carsologica, 29, $123-138$.

Gams, I., 2003: Kras v Sloveniji v prostoru in času. ZRC Publishing, pp. 516, Ljubljana.

Garnier, E., Lavorel, S., Ansquer, P., Castro, H., Cruz, P., Dolezal, J., Erikson, O., Fortunel. C., Freitas, H., Golodets, C., Grigulis, K., Jouany, C., Kazakou, E., Kigel, J., Kleyer, M., Lehsten, V., Lepš, J., Meier, T., Pakeman, R., Papadimitriou, M. Papanastasis, M.P., Quested, H., Quétier, F., Robson, M., Roumet, C., Rusch, G., Skarpe, C., Sternberg, M., Theau, J-P., Thébault, A., Vile, D. \& M.P. Zavarovali, 2007: Assessing the effects of land-use change on plant traits, communities and ecosystem functioning in grasslands: a standardized methodology and lessons from an application to 11 European sites.- Annals of botany, 99, 5, 967-985. DOI: http://dx.doi.org/10.1093/aob/mcl215 
Giusti, C., 2016: Aspects of geodiversity of palaeozoic limestones in the Black mountains of southern France.- Acta geographica Slovenica, 56, 1, 153170. DOI: http://dx.doi.org/10.3986/AGS.963

Gutiérrez F., Parise, M., DeWaele, J. \& H. Jourde, 2014: A review on natural and human-induced geohazards and impacts in karst.- Earth-Science Reviews, 138, 61-88. DOI:10.1016/j.earscirev.2014.08.002

Goslee, S.C. \& M.A. Sanderson, 2010: Landscape context and plant community composition in grazed agricultural systems of the Northeastern United States.Landscape Ecology, 25, 1029-1039. DOI: http:// dx.doi.org/10.1007/s10980-010-9477-y

Gustavsson, E., Lennartsson, T. \& M. Emanuelsson, 2007: Land use more than 200 years ago explains current grassland plant diversity in a Swedish agricultural landscape.- Biological conservation, 138, 1, 47-59.

Hennekens, S.M. \& J.H.J. Schaminée, 2001: TURBOVEG, a comprehensive database management system for vegetation data.- Journal of Vegetation Science, 12, 589-591.

Herbst, C., Wäschke, N., Barto, E. K., Arnold, S., Geuß, D., Halboth, I., Reschke, S., Säger, J., Meiners, T. \& E. Obermaier, 2013: Land use intensification in grasslands: higher trophic levels are more negatively affected than lower trophic levels.- Entomologia Experimentalis et Applicata, 147, 3, 269-281.

Hill, O.H., Roy, D. B. \& K. Thompson, 2002: Hemeroby, urbanity and ruderality: bioindicators of disturbance and human impact.- Journal of Applied Ecology, 39, 5, 708-720. DOI: http://dx.doi.org/10.1046/ j.1365-2664.2002.00746.x

Jóźwiak, M.A. \& M. Jóźwiak, 2014: Bioindication as challenge in modern environmental protection.- Ecological Chemistry and Engineering 21, 4, 577-591. DOI: http://dx.doi.org/10.1515/eces-2014-0041

Kaligarič, M. \& D. Ivajnšič, 2014: Vanishing landscape of the "classic" Karst: changed landscape identity and projections for the future.- Landscape and Urban Planning, 132, 148-158.

Kladnik, D., Kruse, A., B. Komac, 2017: Terraced landscapes: an increasingly prominent cultural landscape type. Acta geographica Slovenica, 57, 2, In Press. DOI: http://dx.doi.org/10.3986/AGS.4770

Klotz, S., Kühn, I. \& W. Durka (eds.), 2002: BIOLFLOR - Eine Datenbank zu biologisch-ökologischen Merkmalen der Gefäßpflanzen in Deutschland. Schriftenreihe für Vegetationskunde 38. Bonn.

Kovačič, G. \& N. Ravbar, 2013: Analysis of human induced changes in a karst landscape: the filling of dolines in the Kras plateau, Slovenia.- Science of total environment, 447, 143-151. DOI: http://dx.doi. org/10.1016/j.scitotenv.2013.01.002
Kogovšek, J. \& M. Petrič, 2013: Increase of vulnerability of karst aquifers due to leakage from landfills.Environmental Earth Sciences, 70, 901-912. DOI: http://dx.doi.org/10.1007/s12665-012-2180-3

Mccune, B. \& M.J. Mefford, 1999: PC-ORD. Multivariate Analysis of Ecological Data, Version 4. MjM Software Design, Glenden Beach.

Merunková, K. \& M. Chytrý, 2012: Environmental control of species richness and composition in upland grasslands of the southern Czech Republic.- Plant Ecology, 213, 4, 591-602.

Mihevc, A., Slabe, T. \& S. Šebela, 1998: Denuded caves an inherited element in the karst morphology, the case from Kras.- Acta Carsologica, 27, 1, 165-174.

Mihevc, A., Prelovšek, M. \& N. Zupan Hajna, 2010: Introduction to the Dinaric Karst, Inštitut za raziskovanje krasa ZRC SAZU, pp 71, Postojna.

Pastor, J., Gutiérrez-Ginés, M.J., Bartolomé, C. \& A.J. Hernández, 2014: The complex nature of pollution in the capping soils of closed landfills: case study in a Mediterranean setting. In: Hernendez-Soriano (ed.) Environmental Risk Assessment of Soil Contamination. InTech, publisher,pp. 199-223, Rijeka.

Peinado, M., Díaz, G., Ocaňa-Peinado, F.M., Aguirre, J.L., Macías, M.A., Delgadillo, J. \& A. Aparicio, 2013: Statistical measure of fidelity applied to diagnostic species in plant sociology.- Modern Applied Science, 7, 6, 106-120. DOI: http://dx.doi. org/10.5539/mas.v7n6p106

Perko, D., Hrvatin, M., R. Ciglič, 2017: Determination of Landscape Hotspots in Slovenia.-Acta geographica Slovenica, 57, 1, 7-29.

Perronne, R., Le Corre, V., Bretagnolle, V. \& S. Gaba, 2014: Stochastic processes and crop types shape weed community assembly in arable fields.- Journal of Vegetation Science, 26, 2, 348-359. DOI: http:// dx.doi.org/10.1111/jvs.12238

Pignatti, S., Menegoni, P. \& S. Pietrosanti, 2005: Bioindicazione attraverso le piante vascolari. Valori di indicazione secondo Ellenberg per le piante della flora d'Italia.- Braun-Blanquetia, 39, 1-97.

Pipan, T. \& D.C. Culver, 2013: Forty years of epikarst: what biology have we learned?.- International journal of speleology, 42, 3, 215-223. DOI: http://dx.doi. org/10.5038/1827-806X.42.3.5.

Pipenbaher, N., Kaligarič, M. \& S. Škornik, 2011: Floristic and functional comparision of karst pastures and karst meadows from the north adriatic karst.- Acta Carsologica, 40, 3, 515-525.

Pleničar, M. \& S. Buser, 1970: Basic Geological map of SFRY. Interpretation of Map L33 - 77, 1 : 100 000.Belgrade. 
Prach, K., 1987: Succession of Vegetation on Dumps from Strip Coal Mining, N. W. Bohemia, Czechoslovakia.- Folia Geobotanica \& Phytotaxonomica, 22, 4, 339-354.

Prach, K. \& P. Pyšek, 2001: Using spontaneous succession for restoration of human-disturbed habitats: Experience from Central Europe.- Ecological Engineering, 17, 55-62. DOI: http://dx.doi.org/10.1016/ S0925-8574(00)00132-4

Prach, K., Pyšek, P. \& V. Jarošík, 2007: Climate and pH as determinants of vegetation succession in Central European man-made habitats.- Journal of Vegetation Science, 18, 5, 701-710. DOI: http://dx.doi. org/10.1111/j.1654-1103.2007.tb02584.x

Prach, K., Lencová, K., Řehounková, K., Dvořáková, H., Jírová, A., Konvalinková, P., Mudrák, A., Novák, J. \& R. Trnková, 2013: Spontaneous vegetation succession at different central European mining sites: a comparison across seres.- Environmental Science and Pollution Research, 20, 11, 7680-7685. DOI: http://dx.doi.org/10.1007/s11356-013-1563-7

Prach, K., Rehounková, K., Lencová, K., Jírová, A., Konvalinková, P., Mudrák, O., Študent, V., Vaněček, Z., Tichý, L., Petřik, P., Šmilauer, P. \& P. Pyšek, 2014: Vegetation succession in restoration of disturbed sites in Central Europe: the direction of succession and species richness across 19 seres.- Applied Vegetation Science, 17, 193-200. DOI: http://dx.doi. org/10.1111/avsc.12064

R Development Core Team, 2015: R: A language and environment for statistical computing. Vienna.

Ravbar, N. \& S. Šebela, 2015: The effectiveness of protection policies and legislative framework with special regard to karst landscapes: Insights from Slovenia.Environmental Science and Policy, 51, 106-116. DOI: http://dx.doi.org/10.1016/j.envsci.2015.02.013

Raunkiaer, C., 1934: The life forms of plants and statistical plant geography.- Charendon Press, pp. 632, Oxford.

Sans, F.X., Armengot, L., Blanco-Moreno, J. M., Bocci, G., Carlesi, S. \& P. Barberi, 2014: Effect of conservation practices on functional diversity and assembly of weed communities: a database of functional traits.- Building Organic Bridges, 3, 977-978.

Sauro, U., 2003: Dolines and dolines: aspects of evolution and problems of classification.- Acta Carsologica, 32, 2, 41-52.

Shushpannikova, G.S., 2014: Formation and degradation of meadows under the impact of hay harvesting and grazing in the Vychegda and Pechora floodplains.Russian journal of ecology, 45, 1, 33-37. DOI: http://dx.doi.org/10.1134/S1067413614010123
Smrekar A., Zorn, M. \& B. Komac, 2016: Heritage protection through a geomorphologist's eyes: from recording to awareness raising.- Acta geographica Slovenica, 56, 1, 123-127. DOI: http://dx.doi. org/10.3986/AGS.3348

Šilc, U. \& A. Čarni, 2007: Formalized classification of the weed vegetation of arable land in Slovenia.- Preslia, 79, 283-302.

Šilc, U., Aćić, S., Škvorc, Ž., Krstonošić, D., Franjić, J. \& Z. Dajić Stevanović, 2014: Grassland vegetation of the Molinio-Arrhenatheretea class in the NW Balkan Peninsula.- Applied vegetation science, 17, 3, 591603. DOI: http://dx.doi.org/10.1111/avsc.12094

Šmid Hribar, M., Geršič, M., Pipan, P., Repolusk, P., Tiran, J., Topole, M., R. Ciglič, 2017: Cultivated terraces in Slovenian landscapes. Acta geographica Slovenica, 57, 2, In Press. DOI: http://dx.doi. org/10.3986/AGS.4597

Sojneková, M. \& M. Chytrý, 2015: From arable land to species-rich semi-natural grasslands: Succession in abandoned fields in a dry region of central Europe.Ecological Engineering, 77, 373-381. DOI: http:// dx.doi.org/10.1016/j.ecoleng.2015.01.042

Tichý, L. \& J. Holt, 2006: JUICE, program for management, analysis and classification of ecological data.Masaryk University, Vegetation Science Group, pp. 98 Brno.

Tichý, L., 2009: Juice, software for vegetation classification.- Journal of Vegetation Science, 13, 3, 451-453. DOI: http://dx.doi.org/10.1111/j.1654-1103.2002. tb02069.x

Tropek, R., Kadlec, T., Karesova, P., Spitzer, L., Kocarek, P., Malenovsky, I., Banar, P., Tuf, I.H., Hejda, M. \& M. Konvicka, 2010: Spontaneous succession in limestone quarries as an effective restoration tool for endangered arthropods and plants.- Journal of Applied Ecology, 47, 139-147. DOI: http://dx.doi. org/10.1111/j.1365-2664.2009.01746.x

Tintner, J. \& B. Klug, 2011: Can vegetation indicate landfill cover features?- Flora- Morphology, Distribution, Functional Ecology of Plants, 206, 6, 559-566. DOI: http://dx.doi.org/10.1016/j.flora.2011.01.005

Tintner, J., Smidt, E., Böhm, K. \& L. Matiasch, 2012: Risk assessment of an old landfill regarding the potential of gaseous emissions - A case study based on bioindication, FT-IR spectroscopy and thermal analysis.Waste management, 32, 2418-2425. DOI: http:// dx.doi.org/10.1016/j.wasman.2012.07.022

Zisenis, M., 2015: Alien plant species: A real fear for urban ecosystems in Europe?- Urban Ecosystems, 18, 355-370. DOI: http://dx.doi.org/10.1007/s11252014-0400-1 